\title{
Intravascular Clearance of Parasitized Erythrocytes in Rodent Malaria
}

\author{
Thomas C. Quinn and DAvid J. Wyler, Laboratory of Parasitic Diseases, \\ National Institute of Allergy and Infectious Diseases, National Institutes of \\ Health, Bethesda, Maryland 20014
}

\begin{abstract}
A B S TRACT Little is known about host defense mechanisms responsible for protective immunity in malaria. The intravascular location of the infection suggested that removal of parasitized erythrocytes by reticuloendothelial organs might be important. To study this possibility, we examined the clearance of ${ }^{51} \mathrm{Cr}$ labeled Plasmodium berghei-infected erythrocytes in rats. Infected erythrocytes were removed more rapidly from circulation than homologous uninfected erythrocytes. The rate of clearance of infected cells during the 1st hour after inoculation was approximately three times greater in rats rendered immune by prior infection than in control rats. This accelerated clearance resulted from greater splenic uptake in immune rats and appeared to correlate with spleen size. Since the clearance pattern of infected erythrocytes more closely resembled the clearance of Heinz body-containing uninfected erythrocytes than of antibody-coated (immunoglobulin G) uninfected erythrocytes, rheologic alterations of parasitized erythrocytes might be a more important determinant of clearance than an antibodydependent process. During the phase of malaria infection in which increasing parasitemia is observed, organ uptake of infected erythrocytes did not increase despite splenic and hepatic enlargement. However during the spontaneous onset of resolution of malaria infection characterized by decreasing parasitemia, a marked enhancement of splenic clearance was noted. These observations suggest that sudden alteration in splenic clearance of parasitized erythrocytes might be important in the resolution of acute malaria.
\end{abstract}

\section{INTRODUCTION}

A major host defense mechanism in intravascular infections is the clearance of microbial pathogens by the reticuloendothelial system (RES). ${ }^{1}$ Factors which regu-

Received for publication 10 October 1978 and in revised form 21 February 1979.

${ }^{1}$ Abbreviations used in this paper: PBS, phosphate-buffered saline; RES, reticuloendothelial system. late clearance include nonspecific trapping of particles in the liver and spleen, and antigen-specific opsonization mediated by antibody (1). The relative role of the RES in antibody-mediated clearance of certain circulating particulate antigens has been shown to be determined primarily by the class of antibody binding the antigen, the density of antibody molecules distributed on the particle, and the binding of complement to the particle (2-6). This type of clearance is largely mediated through specific receptors for immunoglobulin and complement components on the surface of macrophages $(6,7)$. On the other hand, antibody-independent clearance of certain particles depends more upon the regional blood flow through the organs of the RES (8), nonspecific heat-labile opsonins (9-11), and other factors which are poorly understood. The importance of RES clearance as a host defense mechanism is dramatically illustrated in the clinical setting by the overwhelming bacterial sepsis which occurs in some splenectomized individuals (12).

In malaria, the pathogenic stage of the parasite is intraerythrocytic and the infection is confined to the intravascular compartment. Little is known about host defense mechanisms responsible for protective immunity in this disease. The intravascular location of the infection, the markedly deleterious effects of splenectomy on malaria host defense $(13,14)$, and the characteristic histopathology of the disease (15) suggest that RES clearance of infected erythrocytes might be an important host defense mechanism. Several investigators have examined the intravascular clearance of inert particles (colloidal carbon, ${ }^{125} \mathrm{I}$-microaggregated albumin, ${ }^{99 m} \mathrm{Tc}$-sulfur colloid, etc.) in malaria-infected hosts $(16-20)$. These studies have suggested that nonspecific RES clearance is increased in malaria. Although previous studies have measured the survival rate of uninfected erythrocytes in malaria-infected rats (21), no studies have examined in detail the clearance of parasitized erythrocytes. We therefore investigated the role of clearance of Plasmodium berghei-infected erythrocytes in the rat to determine the relative contribution 
of this process to host defense. Our observations suggest that the rheologic rather than the immunological consequences of erythrocyte parasitization are of greatest importance as factors determining clearance of infected erythrocytes. Furthermore, our data indicate that a sudden increase in splenic clearance of parasitized erythrocytes is associated with the onset of spontaneous resolution of acute malaria.

\section{METHODS}

Malaria infections. Male Wistar rats (Hemobartonella free) weighing $125 \mathrm{~g}$ were obtained from Charles River Breeding Laboratories, Wilmington, Mass. Infections were initiated by the intraperitoneal inoculation of $10^{7} P$. berghei (NYU-2 strain)-infected rat erythrocytes suspended in sterile isotonic saline. The level of parasitemia was determined daily by counting the number of parasitized erythrocytes per 1,000 total erythrocytes of Giemsa-stained thin films prepared from tail vein blood.

$P$. berghei infection in adult rats is characterized by the intracellular maturation of the parasite from the ring to the schizont stage. Thereafter, the erythrocyte ruptures, releasing several merozoites which invade other erythrocytes. By day 14 of infection, a peak parasitemia of $40-50 \%$ is attained. The infection then rapidly resolves by crisis, characterized by a sudden fall in parasitemia to undetectable levels by day 20 . For purposes of this study, rats were designated immune $6 \mathrm{wk}$ after resolution of the infection. At this time blood smears were persistently negative for parasites and the rats were resistant to reinfection with the homologous strain of $P$. berghei.

${ }^{51} \mathrm{Cr}$ labeling of $\mathrm{P}$. berghei-infected erythrocytes. Parasitized erythrocytes were obtained by cardiac puncture from infected weanling rats with a $70 \%$ parasitemia. Blood was mixed in $3 \mathrm{vol}$ of citrate-phosphate-dextrose solution $(\mathrm{pH} \mathrm{7.0)}$. The parasitized erythrocytes were separated from uninfected erythrocytes by centrifugation at $900 \mathrm{~g}$ for $5 \mathrm{~min}$ at $20^{\circ} \mathrm{C}$ in capillary tubes. A brownish layer could be readily identified in the cell pellet which contained $85-90 \%$ parasitized erythrocytes. Cells from this layer were labeled with $\mathrm{Na}_{2}{ }^{51} \mathrm{Cr}$ $\mathrm{O}_{4}$ according to previously described methods (22). Briefly, $200 \mu \mathrm{Ci}$ of $\mathrm{Na}_{2}{ }^{51} \mathrm{Cr} \mathrm{O}_{4}(400 \mathrm{mCi} / \mathrm{mg} \mathrm{Cr} \mathrm{sp} \mathrm{act,} \mathrm{Amersham} \mathrm{Corp.,}$ Arlington Heights, Ill.) was added to each milliliter of packed erythrocytes and incubated for $30 \mathrm{~min}$ at $20^{\circ} \mathrm{C}$. Labeled cells were washed three times in isotonic phosphate-buffered saline (PBS, pH 7.2) and resuspended in PBS to a final concentration of $10^{9}$ cells $/ \mathrm{ml}$. $1 \mathrm{ml}$ of the suspension was injected into the tail vein of each rat (weighing $\cong 350 \mathrm{~g}$ ). Normal homologous uninfected rat erythrocytes from control donors were labeled with ${ }^{51} \mathrm{Cr}$ in a similar manner. In preliminary experiments, it was determined that $<2 \%$ of the ${ }^{51} \mathrm{Cr}$ had labeled the parasite themselves. It is known from other studies (23) that ${ }^{21} \mathrm{Cr}$ predominately labels the erythrocyte hemoglobin. Furthermore, $<2 \%$ of the ${ }^{51} \mathrm{Cr}$ was detectable in the plasma at any time after inoculation.

Preparation of Heinz body erythrocytes. Heinz body-containing erythrocytes were prepared by injecting rats subcutaneously with $30 \mathrm{mg} / \mathrm{kg}$ of phenylhydrazine hydrochloride (J. T. Baker Chemical Co., Phillipsburg, N. J.) on two separate occasions, $48 \mathrm{~h}$ apart. Blood was obtained by cardiac puncture and erythrocytes were labeled with ${ }^{51} \mathrm{Cr}$ as described above. To further increase the concentration of Heinz body-containing erythrocytes, the radiolabeled erythrocytes were incubated at $37^{\circ} \mathrm{C}$ for $45 \mathrm{~min}$ in a solution of $10 \mathrm{mM}$ phenylhydrazine (24). The erythrocytes were then washed twice in PBS and resuspended to $10^{9}$ cells $/ \mathrm{ml}$ before inoculation. This prepara- tion consisted of $100 \%$ Heinz body-containing erythrocytes of which $40 \%$ were reticulocytes as determined by examination of blood films stained with $0.5 \%$ methyl violet.

Preparation of antibody-coated rat erythrocytes. Rat erythrocytes were sensitized with rabbit anti-rat erythrocyte antibody. Anti-rat erythrocyte antibody was prepared by injecting $1 \mathrm{ml}$ of an emulsion consisting of a $10 \%$ saline suspension of rat erythrocytes and an equal volume of Freund's complete adjuvant (Difco Laboratories, Detroit, Mich.) into footpads of several New Zealand white rabbits. $3 \mathrm{wk}$ later the rabbits were given a booster injection and were exsanquinated $2 \mathrm{wk}$ thereafter. Because treatment of antiserum with $0.2 \mathrm{M}$ 2-mercaptoethanol had no effect on hemagglutinating or hemolyzing titers (25), anti-rat erythrocyte antibody was presumed to be all of the IgG class (26). To sensitize erythrocytes, washed uninfected rat erythrocytes were labeled with ${ }^{51} \mathrm{Cr}$ and suspended in PBS to a final concentration of $10^{9}$ cells $/ \mathrm{ml}$. Equal volumes of heat-inactivated $\left(56^{\circ} \mathrm{C}\right.$ for $\left.30 \mathrm{~min}\right)$ rabbit antiserum at subhemagglutinating titers $(1: 320)$ were added dropwise to ${ }^{51} \mathrm{Cr}$-labeled erythrocytes with gentle agitation at $37^{\circ} \mathrm{C}$ for $45 \mathrm{~min}$. Cells were washed twice in PBS and suspended at a final concentration of $10^{9} \mathrm{cells} / \mathrm{ml}$ in PBS before infection.

Clearance and organ uptake of ${ }^{51} \mathrm{Cr}$-labeled erythrocytes. In all studies, $1 \mathrm{ml}$ of the prepared suspension containing $10^{951} \mathrm{Cr}$-labeled erythrocytes was injected intravenously into the tail vein of rats. In preliminary clearance experiments with nonimmune rats we determined that this dose resulted in detectable parasites on blood smears, and provided readily detectable levels of ${ }^{51} \mathrm{Cr}$ in small samples of blood. Furthermore, we determined that the clearance pattern of infected erythrocytes was unaffected by the concentration of ${ }^{51} \mathrm{Cr}$-labeled erythrocytes injected.

After infusion, 40- $\mu$ l blood samples were obtained from the retroorbital plexus at regular intervals. The radioactivity present in the plasma and erythrocytes after centrifugation was measured in a Beckman Biogamma Counter (Beckman Instruments, Inc., Cedar Grove, N. J.). The radiolabel remaining in circulation was expressed as percentages of the counts which were present in a sample obtained 1 min after inoculation (designated $100 \%$ ). This amount of radioactivity was not significantly different from the calculated $100 \%$ value based on the blood volume of the rat (total counts injected per rat; $P>0.1$ ). The percentage of ${ }^{51} \mathrm{Cr}$ label remaining in circulation at different times after inoculation was compared for several experiments. The clearance slope was calculated by the method of least squares (27)

To determine organ uptake of ${ }^{51} \mathrm{Cr}$-labeled erythrocytes, different animals were sacrificed at different times after the infusion of ${ }^{51} \mathrm{Cr}$-labeled erythrocytes. Radioactivity was measured in 0.5-g samples of tissue and total organ uptake was calculated based upon the determined wet weight of the organ. Organ uptake was expressed as the percentage of total organ uptake $=($ counts per minute organ $) /($ counts per minute injected) $\times 100$ and as the percentage of organ uptake per gram tissue.

\section{RESULTS}

Clearance and organ uptake of ${ }^{51} \mathrm{Cr}$-labeled erythrocytes. Clearance patterns of uninfected erythrocytes in nonimmune uninfected rats and malaria-immune rats were identical over a 24 -h period (Fig. 1), and no differences in clearance rates were discernable in studies extended over a 3 -wk period $\left(t_{1 / 2}, 16.1 \pm 1.2 \mathrm{~d}\right)$. In contrast, the clearance of infected erythrocytes in both 


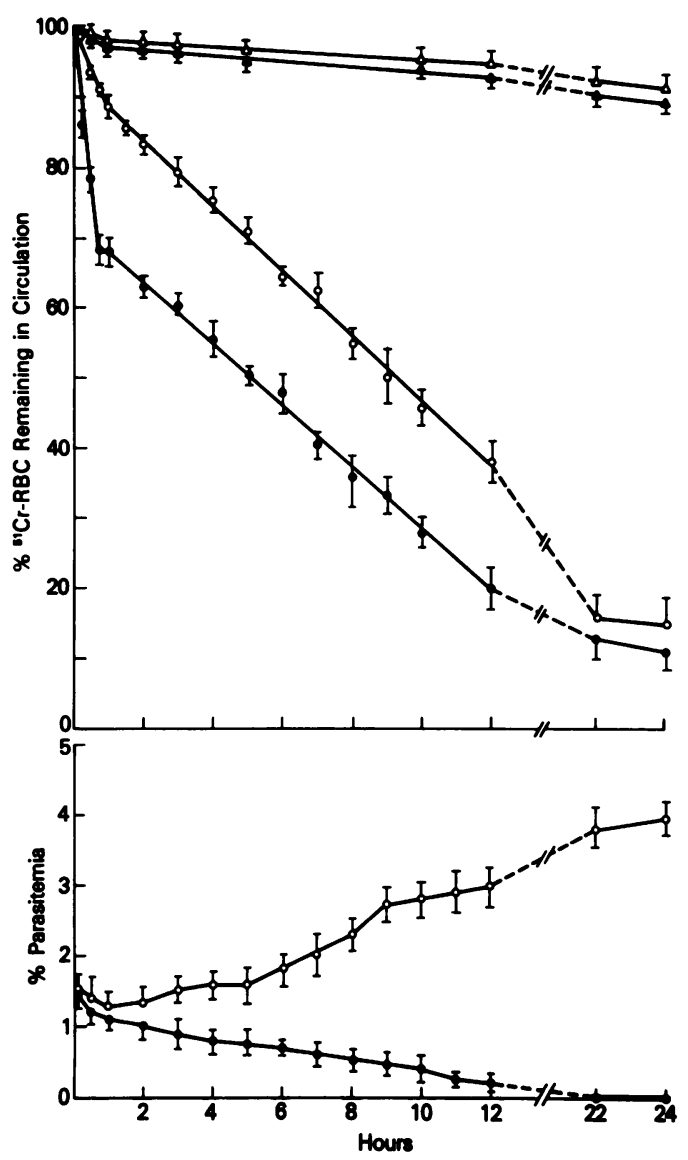

FIGURE 1 Clearance of ${ }^{51} \mathrm{Cr}$-labeled erythrocytes (RBC) after intravenous inoculation in rats immune to malaria $(\Delta, O)$ and in nonimmune rats $(\triangle, O)$. Clearance of uninfected $R B C$ in these rats is represented by triangles; clearance of infected $\mathrm{RBC}$ is represented by circles. Parasitemia (percent infected RBC as determined from blood smears) resulting from the inoculation of ${ }^{51} \mathrm{Cr}$-labeled infected RBC is shown in the lower panel for comparison. Mean \pm SEM, 15 experiments.

nonimmune and immune rats was significantly accelerated ( $t_{1 / 2}$ in nonimmune rats, $9.3 \pm 0.6 \mathrm{~h}$ and $t_{1 / 2}$ in immune rats, $5.0 \pm 0.5 \mathrm{~h} ; P<0.005)$. The pattern of clearance of infected erythrocytes in nonimmune and immune rats was characterized by two distinct phases (Fig. 1). In the 1st h, the clearance rate was rapid in both groups, but was greatest in immune rats $(11.5 \pm 1.5 \%$ of the cells cleared in the nonimmune rats, $32.0 \pm 2.2 \%$ cleared in the immune rats; $P<0.005)$. Disappearance of ${ }^{51} \mathrm{Cr}$ from circulation occurred more gradually during the second phase and at the same rate in both groups. A small percentage (15\%), representing uninfected erythrocytes in the labeled inoculum, persisted in circulation for $>48 \mathrm{~h}$. Parasitemia, as determined from blood smears (Fig. 1), decreased in immune rats at a rate which directly corresponded to the rate of attrition of ${ }^{51} \mathrm{Cr}$-labeled erythrocytes $(r=0.993)$. In contrast, parasitemia in the nonimmune rats continued to rise. Parasite multiplication in these rats was unaffected by prior labeling of infected erythrocytes with ${ }^{51} \mathrm{Cr}$, since the rate of rise of parasitemia was identical to that resulting from inoculation of untreated parasitized erythrocytes.

Labeled erythrocytes were cleared mainly by the spleen and liver; kidney, heart, muscle, bone marrow, lung, and thymus each cleared $<1 \%$ of the inoculum in $24 \mathrm{~h}$. In nonimmune rats, the liver and spleen removed ${ }^{51} \mathrm{Cr}$-labeled erythrocytes at similar low rates (10-15\% of inoculum in each organ) in the first $4 \mathrm{~h}$ after inoculation, but ${ }^{51} \mathrm{Cr}$ continued to accumulate only in the liver (Fig. 2). In contrast, splenic clearance in immune rats was marked during the 1 st h $(34.0 \%$ of inoculum) and continued to increase slightly thereafter. Hepatic clearance in these rats was comparable to that observed in control rats in the first $4 \mathrm{~h}$ but remained relatively constant in the following hours. The greater splenic uptake appeared to be related to the presence of splenomegaly in these animals since splenic uptake per gram tissue was the same for immune and nonimmune rats (Table I).

Effects of splenectomy on clearance of infected erythrocytes. We observed that splenectomy performed $6 \mathrm{wk}$ previously resulted in the abolition of the

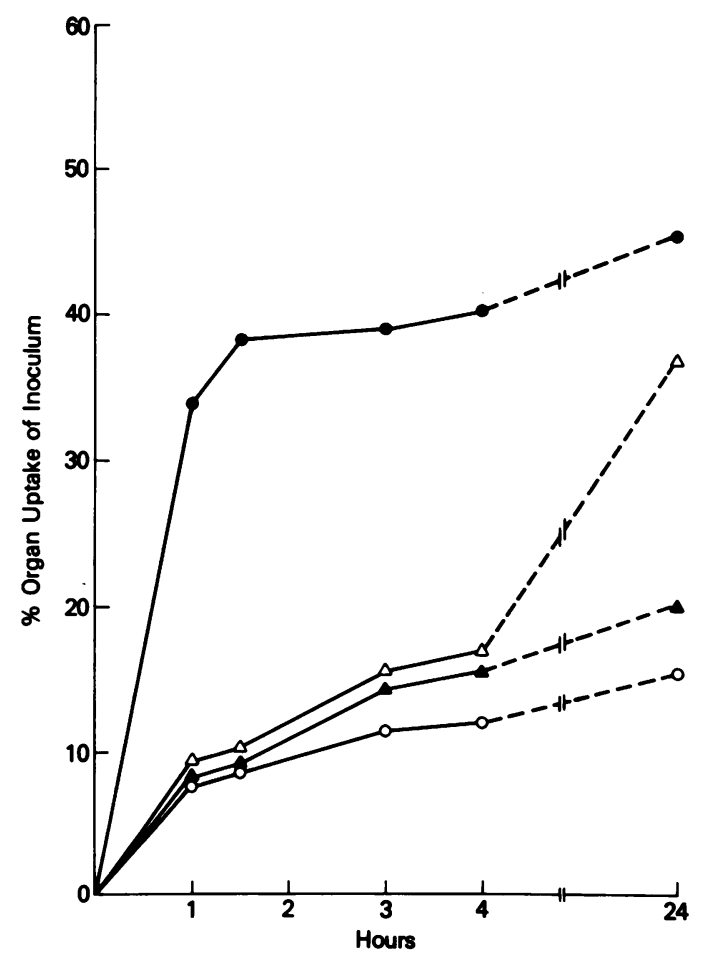

Figure 2 Splenic $(\bullet, O)$ and hepatic $(\Delta, \Delta)$ uptake of ${ }^{51} \mathrm{Cr}$ labeled infected erythrocytes (RBC) after intravenous inoculation in immune rats $(\Theta, \Delta)$ and nonimmune rats $(O, \triangle)$. SEM $<1 \%$ of mean, five experiments. 
TABLE I

$24 h$ Organ Uptake of ${ }^{51} \mathrm{Cr}$-Labeled Infected

Erythrocytes in Intact Rats

\begin{tabular}{lccl}
\hline & Nonimmune & Immune & \multicolumn{1}{c}{$P$} \\
\hline $\begin{array}{l}\text { Organ weight, } g \\
\quad \text { Liver }\end{array}$ & $11.9 \pm 1.73^{*}$ & $13.2 \pm 1.98$ & $>0.1$ \\
$\quad$ Spleen & $1.1 \pm 0.22$ & $2.8 \pm 0.89$ & $<0.005$ \\
Total organ uptake, $\%$ & & & \\
$\quad$ Liver & $36.6 \pm 3.11$ & $19.0 \pm 2.12$ & $<0.005$ \\
$\quad$ Spleen & $16.1 \pm 0.88$ & $46.4 \pm 1.31$ & $<0.005$ \\
Organ uptake/gram & & & \\
$\quad$ tissue, $\$$ & & & \\
$\quad$ Liver & $3.0 \pm 0.38$ & $1.4 \pm 0.19$ & $<0.005$ \\
$\quad$ Spleen & $14.6 \pm 1.38$ & $16.5 \pm 1.62$ & $>0.1$ \\
\hline
\end{tabular}

* All data expressed as mean $\pm \mathrm{SE}$.

$\$$ Percentage of total organ uptake $=$ (counts per minute organ)/(counts per minute injected) $\times 100$.

$\S$ Percentage of organ uptake per gram tissue $=$ total organ uptake/weight of the organ (gram).

early (1st $h$ ) rapid phase in both the immune and nonimmune rats (Fig. 3). The clearance rates were the same in both groups of splenectomized animals $(P$ $>0.1$ ) and were similar to the rate of the second phase of clearance observed in intact rats (slope in splenectomized rats, -0.78 ; slope of second phase in intact rats, $-0.93 ; P<0.05$, Student's $t$ test). Parasitemia rose in splenectomized nonimmune rats but remained constant in splenectomized immune rats (Fig. 3 ).

Clearance of antibody-coated and Heinz body-containing erythrocytes. We attempted to determine whether differences in clearance patterns in immune and nonimmune rats related to a greater ability of immune spleens to remove antibody-coated or rheologically altered erythrocytes. Rat erythrocytes coated with large subhemagglutinating concentrations of rabbit anti-rat erythrocyte antibody (immunoglobulin $G$ ) were cleared at similar rates in both groups $\left(t_{1 / 2}\right.$ in nonimmune rats, $0.75 \mathrm{~h} ; \mathrm{t}_{1 / 2}$ in malaria-immune rats, $1.5 \mathrm{~h}$ ) (Fig. 4A). Organ uptake was also similar in both groups (Table II). Splenectomy had a comparable effect in decreasing the clearance of sensitized erythrocytes in immune and nonimmune rats. It therefore seemed unlikely that the more extensive clearance of infected erythrocytes in immune rats related to an increased ability of their spleen to remove erythrocytes coated with IgG. In contrast, clearance of Heinz body-containing erythrocytes was greater in immune rats than in nonimmune rats and was entirely spleen dependent (Fig. 4B, Table II). These erythrocytes share with parasitized erythrocytes the property of decreased deformability $(28,29)$ and the presence of a large intracellular inclusion. It thus appeared that the immune rat differed from the nonimmune rat by the greater ability of the immune spleen to remove rheologically altered erythrocytes.

Alteration of clearance during the course of malaria infection. Because the above studies were performed on rats without detectable parasitemia, we examined the role of clearance during active infection. We infected 30 nonimmune rats with $10^{7}$ infected erythrocytes and clearance studies were performed on different groups of rats at various times during the infection. The clearance patterns on days $4,7,11$, and 14 of infection (precrisis period associated with increasing parasitemia) were all the same $(P>0.1, F$ test of slope) and was characterized by a monophasic clearance (Fig. 5 ). This monophasic pattern resembled that observed in uninfected splenectomized rats (Fig. 3). Although spenomegaly developed during this period, splenic uptake remained constant and hepatic uptake progressively

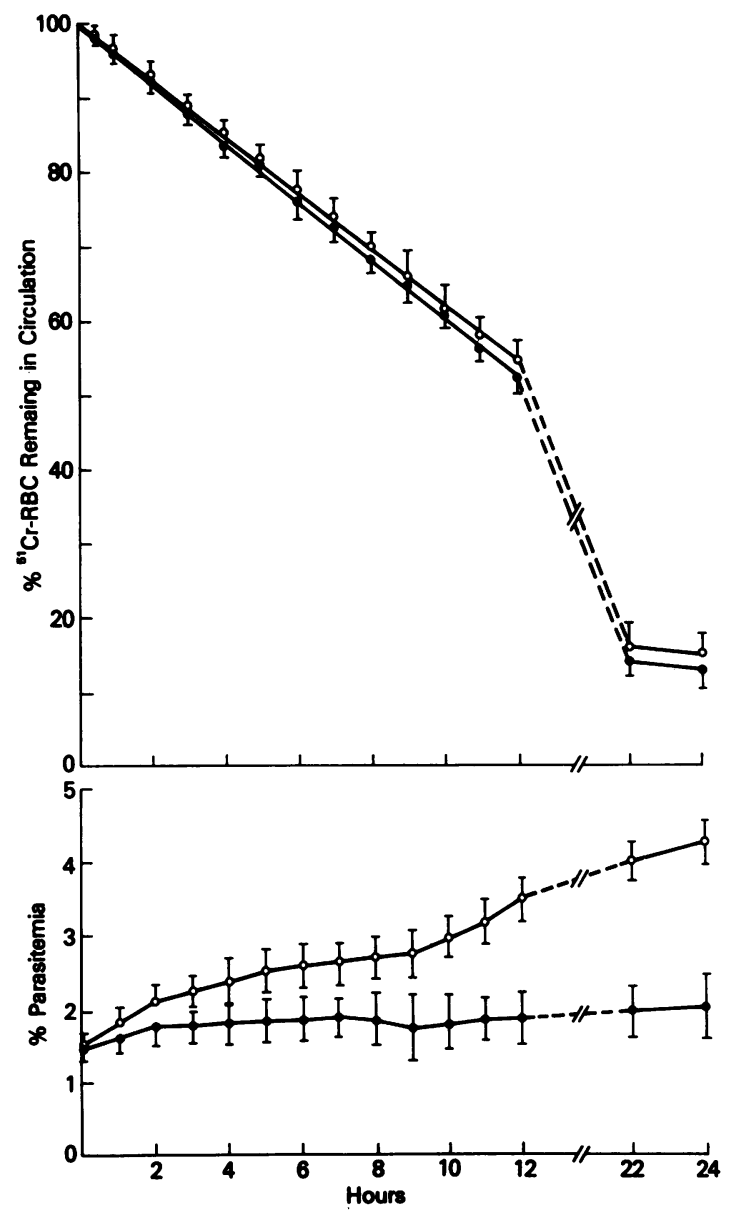

FIGURE 3 Clearance of ${ }^{51} \mathrm{Cr}$-labeled infected erythrocytes (RBC) in splenectomized immune (O) and nonimmune $(O)$ rats. Parasitemia (percent infected RBC as determined from blood smears) resulting from the inoculation of ${ }^{51} \mathrm{Cr}$-labeled infected RBC is shown in the lower panel for comparison. Mean \pm SEM, 10 experiments. 

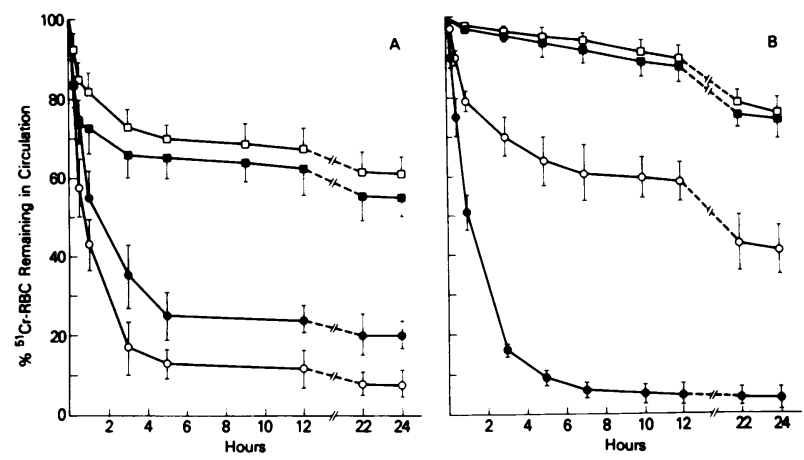

FIGURE 4 Clearance of ${ }^{51} \mathrm{Cr}$-labeled uninfected erythrocytes (RBC) sensitized with rabbit anti-rat RBC antibody (A) or containing Heinz bodies $(B)$ in malaria-immune $(\boldsymbol{O}, \mathbf{\square})$ and nonimmune rats $(O, \square)$. Intact rats are represented by circles; splenectomized rats are represented by squares. Mean $\pm S E M$, five experiments.

decreased below control levels (Fig. 6). In marked contrast, clearance during the crisis period (days 17-19, at a time when parasitemia fell) was biphasic and dramatically accelerated (Fig. 5). This increased clearance was associated with a sudden ability of the spleen, but not the liver, to remove ${ }^{51} \mathrm{Cr}$-labeled infected erythrocytes. This dramatic change in splenic uptake was not associated with a sudden increase in spleen size between the immediate precrisis and crisis period (Fig. 6).

\section{DISCUSSION}

In this investigation of clearance of $P$. berghei-infected erythrocytes, we determined that infected erythrocytes were removed more rapidly from circulation than homologous uninfected erythrocytes (Fig. 1). This accelerated clearance was more pronounced in rats immune to $P$. berghei malaria than in nonimmune uninfected or infected precrisis rats (Figs. 1 and 5). The accelerated clearance was primarily dependent upon

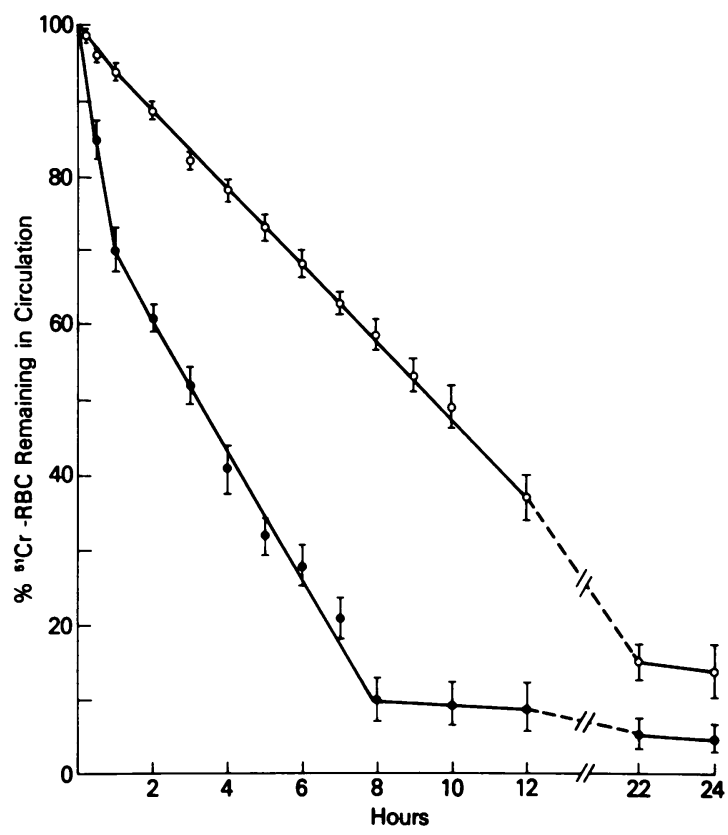

FIGURE 5 Clearance of ${ }^{51} \mathrm{Cr}$-labeled erythrocytes (RBC) in rats on days $4,7,11,14(\bigcirc)$ and days 17 and $19(O)$ of infection with $P$. berghei. Clearance rates did not differ on different days of infection within each group $(P>0.1)$. Mean \pm SEM, 10 experiments.

splenic uptake (Fig. 2, Table I) and all differences in clearance rates were abolished by splenectomy (Fig. 3). The greater splenic uptake in immune rats was directly associated with the presence of splenomegaly in these animals (Table I). This pattern of clearance of infected erythrocytes more closely resembled that of Heinz body-containing erythrocytes than that of IgG-coated uninfected erythrocytes (Fig. 4). These observations suggested that the accelerated clearance of parasitized erythrocytes might be based on alterations in the rheologic properties of these cells rather than an anti-

TABLE II

24 h Organ Uptake of ${ }^{51} \mathrm{Cr}$-Labeled Antibody-Coated Erythrocytes and ${ }^{51} \mathrm{Cr}$-Labeled Heinz Body-Containing Erythrocytes*

\begin{tabular}{|c|c|c|c|c|c|c|}
\hline & \multicolumn{2}{|c|}{ Organ weight } & \multicolumn{2}{|c|}{$\begin{array}{l}\text { Antibody-coated } \\
\text { erythrocytes }\end{array}$} & \multicolumn{2}{|c|}{$\begin{array}{l}\text { Heinz body-containing } \\
\text { erythrocytes }\end{array}$} \\
\hline & Nonimmune & Immune & Nonimmune & Immune & Nonimmune & Immune \\
\hline \multicolumn{7}{|l|}{ Intact rats } \\
\hline Liver & $11.5 \pm 2.13 \ddagger$ & $13.6 \pm 2.12$ & $34.7 \pm 2.31$ & $31.1 \pm 1.87$ & $19.7 \pm 1.91$ & $20.1 \pm 2.31$ \\
\hline Spleen & $1.2 \pm 0.34$ & $2.9 \pm 1.01 \S$ & $42.3 \pm 3.21$ & $35.6 \pm 2.89$ & $29.3 \pm 3.21$ & $61.3 \pm 5.61 \S$ \\
\hline \multicolumn{7}{|l|}{ Splenectomized rats } \\
\hline Liver & $12.8 \pm 2.12$ & $13.8 \pm 2.49$ & $44.5 \pm 1.68$ & $48.4 \pm 3.22$ & $29.1 \pm 2.05$ & $37.3 \pm 3.21 \S$ \\
\hline
\end{tabular}

$*$ Percentage of organ uptake $=($ counts per minute organ $) /($ counts per minute injected $) \times 100$.

$\ddagger$ All data expressed as mean $\pm \mathrm{SE}$.

$\S P<0.005$. 


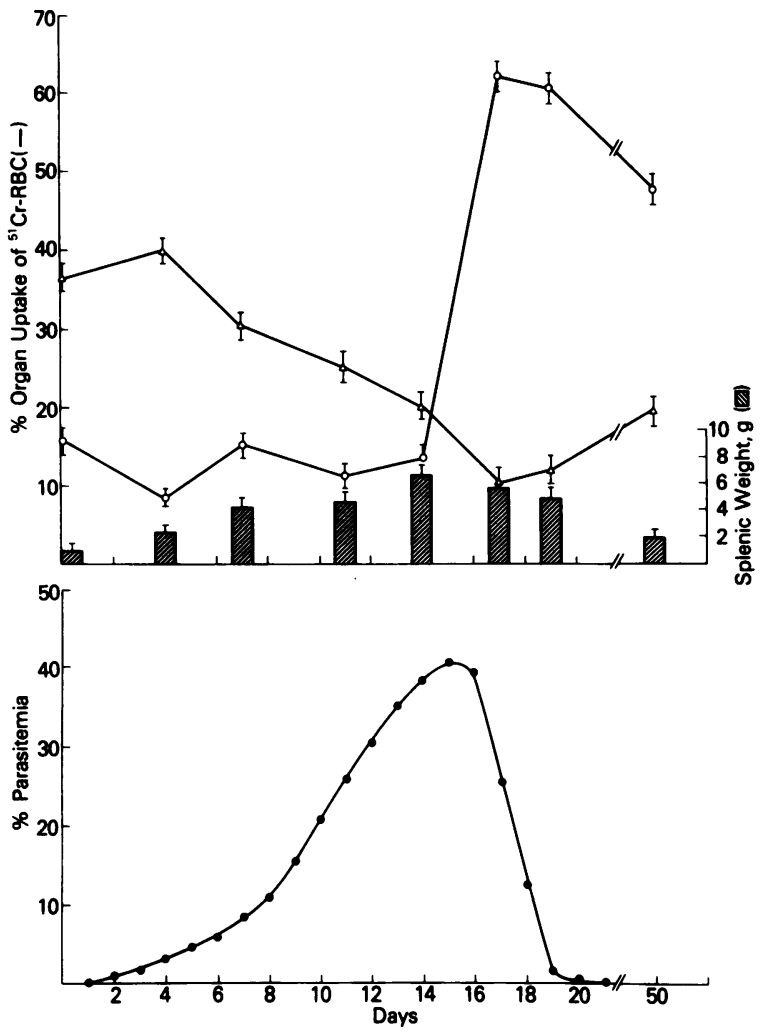

Figure 6 Splenic $(O)$ and hepatic $(\triangle)$ uptake of ${ }^{51} \mathrm{Cr}$-labeled $P$. berghei-infected erythrocytes (RBC) in rats on different days of infection with $P$. berghei. Spleen weight on corresponding days of infection is shown by vertical bars. Mean parasitemia (percent infected RBC) on each day of infection is shown for comparison in the lower panel (SEM $<1 \%$ of mean). The crisis period occurred between days 16 and 19 of infection.

body (IgG)-dependent process. In addition, since we observed that splenic clearance suddenly increased in infected rats at the time of crisis (Figs. 5 and 6), splenic clearance may be important as a host defense mechanism in the resolution of acute malaria infection.

A striking feature in the clearance of infected erythrocytes was the biphasic pattern in the disappearance of ${ }^{51}$ Cr-labeled cells from circulation (Fig. 1). The first phase, which occurred within the 1 st $h$ after inoculation in both immune and nonimmune rats, was characterized by a rapid state of clearance which was most pronounced in immune rats. On the basis of organ uptake of ${ }^{51} \mathrm{Cr}$ (Fig. 2) and the effect of splenectomy (Fig. 3), it was possible to determine that this rapid first phase represented splenic uptake. The slower second phase (Fig. 1) was the same in both immune and nonimmune rats. Presumably this represented the disappearance of ${ }^{51} \mathrm{Cr}$ from circulation as a result of sequestration of labeled erythrocytes containing schizonts (mature parasites) in the deep vascular beds (30), their subse- quent rupture, and the rapid renal excretion of the released ${ }^{51} \mathrm{Cr}$ (23). The gradual rather than precipitous decline in circulating ${ }^{51} \mathrm{Cr}$ resulted from the asynchronous maturation of $P$. berghe $i$ (31) culminating in schizont rupture, invasion of unlabeled erythrocytes by merozoites, and rising parasitemia. This was reflected by the observation that the rate of increase of parasitemia in nonimmune rats was inversely proportional $(r$ $=0.995$ ) to the rate of decline of ${ }^{51} \mathrm{Cr}$ in the second phase (Fig. 1).

Spleen size was a major determinant of the magnitude of clearance of infected erythrocytes and Heinz body-containing erythrocytes, but not antibody-coated erythrocytes (Tables I and II). In related studies we determined that splenomegaly induced by stimuli other than malaria, such as by the repeated intraperitoneal injections of methyl cellulose (32), were equally effective in accelerating the clearance of parasitized erythrocytes. ${ }^{2}$ Furthermore, the magnitude of splenic clearance was observed to be directly related to spleen weight $(r=0.92)$ when studies were performed in rats with methyl cellulose-induced or malaria-induced splenomegaly of different magnitude (data not shown). These observations suggested that splenomegaly per se, rather than a specific antiplasmodial alteration in splenic function, was the basis for the accelerated clearance in immune rats. The greater ability of enlarged spleens to remove infected erythrocytes is likely to depend upon the increased efficiency of the hypertrophied organ to remove poorly deformed erythrocytes (33). Support for this notion derives from our observations that another poorly deformable erythrocyte, the Heinz body-containing cell, is also cleared from circulation more readily in the setting of splenomegaly, whether induced by malaria (Fig. 4B) or methyl cellulose treatment (data not shown). The more extensive removal of Heinz body-containing erythrocytes than infected erythrocytes might therefore represent quantitative differences in altered deformability of these two erythrocyte populations.

In contrast to the above evidence suggesting an important role of the rheological properties of infected erythrocytes in their removal from circulation, we have found no evidence that antibody-mediated clearance of these cells occurs. That is, in related studies ${ }^{3}$ we have determined that although hyperimmune serum can transfer to nonimmune rats protective immunity to $P$. berghe $i$, it fails to significantly alter the pattern of clearance of splenic uptake of infected erythrocytes in the recipients. The failure to find evidence for the occurrence of opsonization of intact infected erythrocytes in vivo is not incompatible with the observation that parasites removed artificially from their intracel-

\footnotetext{
${ }^{2}$ Quinn, T., and D. Wyler. Unpublished observations.

${ }^{3}$ Quinn, T., and D. Wyler. Manuscript in preparation.
} 
lular location can be opsonized in vivo (34). This suggests that malaria parasites may be protected from a potentially important host defense mechanism by virtue of their intracellular location and the lack of expression of parasite antigen on the surface of the infected erythrocyte (35). Antiplasmodial antibody may therefore act upon the extracellular merozoite after schizont rupture and thus prevent invasion of other erythrocytes (36).

In view of these considerations of the clearance of parasitized erythrocytes, it was surprising to find a sudden increase in splenic clearance at the time of crisis (Fig. 6). The increase clearance was not associated with a sudden increase in spleen size during infection. Moreover, it followed a precrisis period in the infection during which the efficiency of splenic clearance (i.e., organ uptake per gram tissue) was below control levels. The precise explanation for the sudden increase in clearance is not available from our data. One possibility which we are presently investigating is that these changes represent sudden alteration in splenic microcirculation allowing for greater flow through the "open" pathways. The open pathway of circulation brings erythrocytes into contact with narrow fenestrations in the splenic cords which might result in extensive trapping of poorly deformable cells $(37,38)$. In addition, passage of erythrocytes through the cords would bring them into close contact with cordal macrophages, cells which might have been suggested to be important in mediating a process of killing of intraerythrocytic parasites without phagocytosis (39).

Thus, it appears from our data that splenic clearance of infected erythrocytes might be an important mechanism of host defense at the time of crisis when resolution of the acute infection occurs. Indeed, we have observed in related studies that the induction and maintenance of crisis is critically dependent upon the continuous presence of the spleen. ${ }^{3}$ However, the ability of immune rats to resist rechallenge with $P$. berghei appears to depend upon mechanisms which are largely spleen-independent. Parasitemia in splenectomized immune rats did not rise after rechallenge (Fig. 3), in contrast to the rising parasitemia observed in intact and splenectomized nonimmune rats. Antiplasmodial defense in immune rats is incompletely understood but may depend upon antibody-mediated inhibition of merozoite invasion $(36,40)$. These findings suggest that different mechanisms of host defense might be important in resolution of acute primary infections and resistance to rechallenge in immune rats.

\section{ACKNOWLEDGMENTS}

The authors thank Doctors M. Frank, L. Miller, and I. Green for their helpful suggestions in the preparation of this manuscript, and we gratefully acknowledge the secretarial assistance of M. Nicholson.

\section{REFERENCES}

1. Saba, T. M. 1970. Physiology and physiopathology of the reticuloendothelial system. Arch. Intern. Med. 126: 1031- 1052 .

2. Jandl, J. H., A. R. Jones, and W. B. Castle. 1957. The destruction of red cells by antibodies in man. I. Observations on the sequestration and lysis of red cells altered by immune mechanisms. J. Clin. Invest. 36: 1428-1459.

3. Jandl, J. H., and M. E. Kaplan. 1960. The destruction of red cells by antibodies in man. III. Quantitative factors influencing the patterns of hemolysis in vivo. J. Clin. Invest. 39: 1145-1156.

4. Spiegleberg, H., P. Miescher, and B. Benacerraf. 1963. The role of complement in the immune clearance of rat erythrocytes and E. coli by the RES in mice.J. Immunol. 90: 751-759.

5. Schulkind, M. L., E. F. Ellis, and R. T. Smith. 1967. Effect of antibody upon clearance of ${ }^{125}$ I-labeled pneumococci by the spleen and liver. Pediatr. Res. 1: 178-184.

6. Frank, M. M., A. D. Schreiber, J. P. Atkinson, and C. J. Jaffee. 1977. Pathophysiology of immune hemolytic anemia. Ann. Intern. Med. 87: 210-222.

7. Huber, H., M. J. Polley, W. D. Linscott, H. H. Fudenberg, and H. J. Muller-Eberhard. 1968. Human monocytes: distinct receptor sites for the third component of complement and for immunoglobulin G. Science (Wash. D. C.). 162: 1281-1283.

8. Dobson, E. L. 1957. Factors controlling phagocytosis. In Physiopathology of the Reticuloendothelial System. B. N. Halpern, editor. Charles C Thomas, Publisher, Springfield, Ill. 80-114.

9. Rowley, D. 1960. The role of opsonins in nonspecific immunity. J. Exp. Med. 111: 137-144.

10. Jenkin, C. R., and D. Rowley. 1961. The role of opsonins in the clearance of living and inert particles by the cells of the reticuloendothelial system. J. Exp. Med. 114: 363-374.

11. Saba, T. M. 1974. Aspecific opsonins. In The Immune System and Infectious Diseases. S. Karger AG, Basel, Switzerland. 489-504.

12. Singer, D. B. 1973. Postsplenectomy sepsis. In Perspectives in Pediatric Pathology. H. S. Rosenberg and R. P. Bolande, editors. Yearbook Medical Publishers, Inc. 1: 285-311.

13. Garnham, P. C. C. 1970. The role of the spleen in protozoal infections with special reference to splenectomy. Acta Trop. 27: 1-13.

14. Wyler, D. J., C. N. Oster, and T. C. Quinn. 1979. In The Role of the Spleen in the Immunology or Paracitic Diseases. Tropical Disease Research Series No. 1. Schwabe $\&$ Co., Basel. In press.

15. Taliaferro, W. H., and H. W. Mulligan. 1937. The histopathology of malaria, with special reference to the function and origin of the macrophages in defense. Indian Med. Res. Mem. 29: 1-138.

16. Lucia, H. L., and R. S. Nussenzweig. 1969. Plasmodium chabaudi and Plasmodium vinckei: phagocytic activity of mouse reticuloendothelial system. Exp. Parasitol. 25: 319-323.

17. Sheagren, J. N., J. E. Tobie, L. M. Fox, and S. M. Wolff. 1970. Reticuloendothelial system phagocytic function in naturally acquired human malaria. J. Lab. Clin. Med. 75: 481-487.

18. Kitchen, A. G., and N. R. DiLuzio. 1971. Influence of Plasmodium berghei infections on phagocytic and humoral recognition factor activity. J. Reticuloendothel. Soc. 9: 237-247.

19. Loose, L. O., and N. R. DiLuzio. 1976. A temporal relation- 
ship between reticuloendothelial system phagocytic alterations and antibody responses in mice infected with Plasmodium berghei (NYU-2 Strain). Am. J. Trop. Med. Hyg. 25: 221-228.

20. Ziessman, H. A. 1976 . Lung uptake of ${ }^{99 \mathrm{~m}}$ Tc-sulfur colloid of falciparum malaria case report. J. Nucl. Med. 171: 794-796.

21. George, J. N., E. F. Stockes, O. J. Wicker, and M. E. Conrad. 1966. Studies of the mechanism of hemolysis in experimental malaria. Mil. Med. 131: 1217-1224.

22. Mollison, P. L. 1972. Blood transfusion in Clinical Medicine. 5th edition. Blackwell Scientific Publications Ltd. Oxford.

23. Ebaugh Jr., F. G., C. P. Emerson, and J. F. Ross. 1953. The use of radioactive chromium 51 as an erythrocyte tagging agent for the determination of red cell survival in vivo. J. Clin. Invest. 32: 1260-1276.

24. Klausner, M. A., L. J. Hirsch, P. F. Lebland, J. K. Chamberlain, M. R. Klemperer, and G. B. Segel. 1975. Contrasting splenic mechanisms in the blood clearance of red blood cells and colloidal particles. Blood. 46: 965-976.

25. Kabat, E. A., and M. M. Mayer. 1971. Experimental Immunochemistry. 2nd edition. Charles C Thomas, Publisher, Springfield, Ill. 150-151.

26. Deutsch, H. F., and J. I. Morton. 1957. Dissociation of human serum macroglobulins. Science (Wash. D. C.). 125: $600-601$.

27. Dixon, W. J., and F. J. Massey, Jr. 1969. Introduction to Statistical Analysis. McGraw-Hill Book Co. New York. $198 \mathrm{pp}$.

28. Miller, L. H., S. Usami, and S. Chen. 1971. Alteration in the rheologic properties of Plasmodium knowlesi-infected red cells. A possible mechanism for capillary obstruction. J. Clin. Invest. 50: 1451-1455.
29. Lubin, A., and J. F. Desforges. 1972. Effect of Heinz bodies on red cell deformability. Blood. 39: 658-665.

30. Alger, N. E. 1963. Distribution of schizonts of Plasmodium berghei in tissues of rats, mice and hamsters. J. Protozool. 10: 6-10.

31. Garnham, P. C. C. 1966. Malaria Parasites and Other Haemosporidia. Blackwell Scientific Publications Ltd., Oxford.

32. Palmer, J. G., E. J. Eichwald, G. E. Cartwright, and M. M. Wintrobe. 1953. The experimental production of splenomegaly, anemia, and leukopenia in albino rats. Blood. 8: 72-80.

33. Wagner, H. N., M. A. Razzak, R. A. Gaertner, W. P. Caine, and O. T. Feagin. 1962. Removal of erythrocytes from the circulation. Arch. Intern. Med. 110: 128-135.

34. Hamburger, J., and J. P. Kreier. 1975. Antibody-mediated elimination of malaria parasites (Plasmodium berghei) in vivo. Infect. Immun. 12: 339-345.

35. Howard, R. J., and G. F. Mitchell. 1978. External Proteins on hemosporidial infected mouse erythrocytes. $J$. Supramol. Struc. 2(Suppl.): 218. (Abstr.)

36. Miller, L. H., M. Aikawa, and J. A. Dvorak. 1975. Malaria (Plasmodium knowlesi) merozoites: immunity and the surface coat. J. Immunol. 114: 1237-1242.

37. Weed, R. I. 1970. The importance of erythrocyte deformability. Am. J. Med. 49: 147-150.

38. Rifkind, R. A. 1966. Destruction of injured red cells in vivo. Am. J. Med. 41: 711-723.

39. Allison, A. C., and I. A. Clark. 1977. Specific and nonspecific immunity to haemoprotozoa. Am. J. Trop. Med. Hyg. 26: 216-222.

40. Cohen, S., G. A. Butcher, and R. B. Crandall. 1969. Action of antimalarial antibody. Nature (Lond.). 223: 368-371. 